\title{
Influence of steam heat transfer tube damage characteristics on burst pressure
}

\author{
Zhizhen Peng ${ }^{1, a}$, Shangkun Ren ${ }^{2}$ \\ ${ }^{1}$ Suzhou Nuclear Power Research Institute Co., Ltd., Suzhou 215000, Jiangsu China \\ ${ }^{2}$ Key Laboratory of Non-destructive Testing of Ministry of Education, Nanchang Hangkong University Nanchang 330063, China
}

\begin{abstract}
The corrosion damage and leakage of heat transfer tube of steam generator were important factors of nuclear power accident, which is closely related to the national nuclear power production safety and people's life and property. The influence of the size change of cylindrical defect on burst pressure of inconel690 alloy heat transfer tube was studied by finite element simulation and experimental measurement. According to the simulation and test data, the relationship between burst pressure and damage parameter is established. The results show that for cylindrical defects with small depth, there is an extreme point of cylinder damage diameter $\mathrm{b}$ which makes the damage maximum, which makes the burst pressure at a minimum. The research results can provide reference for accurate evaluation of residual life of heat transfer tubes.
\end{abstract}

\section{Introduction}

The safety of heat transfer tube of steam generator is an important problem of nuclear power structure integrity. Steam generator tubes shall be designed and used to prevent leakage of primary water containing radioactive materials $[1,2]$. Therefore, regular in-service inspection is required. When cracks are found, fracture mechanism and damage degree evaluation are needed to determine whether to continue to use or repair. In order to fracture mechanics evaluation, the burst (maximum allowable) pressure of the steam generator tube must be estimated $[3,4]$. At present, there are few studies on the effect of surface damage size on burst pressure [5]. There is no reliable and scientific assessment method for residual life of damage $[6,7]$.

At present, corrosion and wear are the main degradation types of steam generator tubes in most nuclear power plants. With the increase of operation time, the wear of wall thickness with thinning amount $\geq$ $10 \%$ increases. In this paper, based on the internal structure characteristics of steam generator, a group of artificial cylindrical damage forms with different sizes can be used to describe the common degradation mechanism of heat transfer tubes. The relationship between the size characteristics of artificial injury and burst pressure is studied, and the comparison with the simulation data is carried out to provide reference for accurate evaluation of residual life of heat transfer tubes.

\section{Finite element analysis of effect of heat transfer tube damage characteristics on burst pressure}

The final printed area will be $172 \times 252 \mathrm{~mm}$. The commonly methods used to simulate the bursting pressure of heat transfer tube are experimental analysis method, plastic analysis method and limit analysis method. Based on the principle of limit analysis and numerical simulation method, the influence of corrosion defect characteristics on burst strength of steam generator heat transfer tube was analyzed. According to the geometric size of the heat transfer tube, the burst pressure of the heat transfer tube was obtained by limit analysis method.

The finite element analysis software is ANSYS Workbench, which adopts the multivariate finite element model. The model has various calculation types, rich models and high accuracy.

Model parameters: the material of heat transfer tube of steam generator is Inconel690 alloy material, the size is $\Phi 19.05 \mathrm{~mm} \times 1.09 \mathrm{~mm}$, and the relevant parameters are shown in Table 1 .

Table 1. Related parameters of Inconel690 alloy.

\begin{tabular}{|c|c|}
\hline Tube material & Inconel690 \\
\hline Density & $8190 \mathrm{~kg} / \mathrm{m}^{3}$ \\
\hline Elastic modulus & $211 \mathrm{GPa}$ \\
\hline Poisson's ratio & 0.289 \\
\hline Yield strength & $239 \mathrm{MPa}$ \\
\hline
\end{tabular}

\footnotetext{
a pengzhizhen@cgnpc.com.cn
} 


\begin{tabular}{|c|c|}
\hline Tensile strength & $570 \mathrm{MPa}$ \\
\hline Flow stress & $404.5 \mathrm{MPa}$ \\
\hline
\end{tabular}

Inconel690 alloy is a nonlinear material with ultrahigh toughness. The main failure mode is plastic failure. In this paper, the finite element simulation software is used to analyze the limit of heat transfer tubes with cylindrical defects (corrosion pits) and calculate the burst pressure of heat transfer tubes.

\subsection{Setting of pre-processing module, solution and post-processing module}

In the pre-processing module, the element type is solid185, which has eight nodes, and each node has three degrees of freedom, and the node coordinate system is translational in $\mathrm{x}, \mathrm{y}, \mathrm{z}$ directions. This element has the functions of plasticity, hyperelasticity, stress stiffening, creep, large deformation and large strain.

The simplified model was established: because of the symmetry of heat transfer tube structure and load, the model was sliced and $1 / 4$ of the model was studied. In order to prevent the uneven stress distribution of the model port, a spherical head is added at both ends of the model. The length of the model is $150 \mathrm{~mm}$, which meets the requirements of Saint Venant's theorem.

Model discretization: in order to save calculation cost and improve calculation accuracy, global and local mesh generation is adopted.

The ideal elastic-plastic material model is adopted, and the real yield strength is taken as the yield strength of the ideal material without considering the strain strengthening effect of the material. The flow stress represents the yield limit under the strain rate, and its value directly affects the solution of burst pressure. The average value of yield limit and strength limit is taken in the simulation.

In the solution setting operation, the boundary conditions are as follows: the shape of the pipe and the corrosion defect is regular and completely symmetrical, and the symmetrical constraint is applied to both ends of the pipe, and the symmetrical constraint is also applied to the axial split pipe wall, so as to avoid the generation of bending moment load.

Load setting: only considering the change of pipe burst pressure under internal pressure, ignoring the effect of other loads on the pipe wall. The analysis settings are as follows: $100 \mathrm{MPa}$ load is applied on the inner wall of heat exchange tube, which is decomposed into several load sub steps. Open the automatic time step and set the sub load step. The size of the sub step and the thickness of the mesh jointly determine the accuracy of the final bursting pressure. Open the large deformation, close the weak elasticity, and set the stabilization of the nonlinear part as a constant.

Solution of burst pressure: ANSYS uses Newton Lapson equilibrium iteration to solve nonlinear problems, forcing the solution to reach equilibrium convergence (within a certain tolerance range) at the end of each load increment. If the convergence criterion is not satisfied, the non-equilibrium load is re estimated and the stiffness matrix is modified to obtain a new solution. If a pressure of $100 \mathrm{MPa}$ is applied to the pipeline, the solution will not converge if the pressure is too large. The pressure load of a sub step before convergence is the burst pressure.

Setting of post-processing module: the ANSYS postprocessing module can easily observe the relationship between the applied pressure load and the maximum displacement by inserting a chart, as well as the corresponding table of load step, solution time and pressure value. In the process of simulation, the simulation value is modified according to the test data to keep the change rule of simulation data stable. The simulation results of bursting pressure of heat transfer tube are shown in Table 2.

\subsection{Damaged heat transfer tube model of simulation and test}

Taking the common form of pit damage of heat transfer tube as an example, the depth of cylindrical pit is a and the diameter of cylindrical pit is $b$. the specific processing scheme and finished product drawing of artificial injury of sample are shown in Fig. 1. Fig. 1a shows the schematic diagram of heat transfer tube with pit defect, and fig. $1 \mathrm{~b}$ shows the finished product drawing of heat transfer tube with pit defect. Table 2 shows the simulation data of $350{ }^{\circ} \mathrm{C}$ burst pressure of heat transfer tube with pit defect.

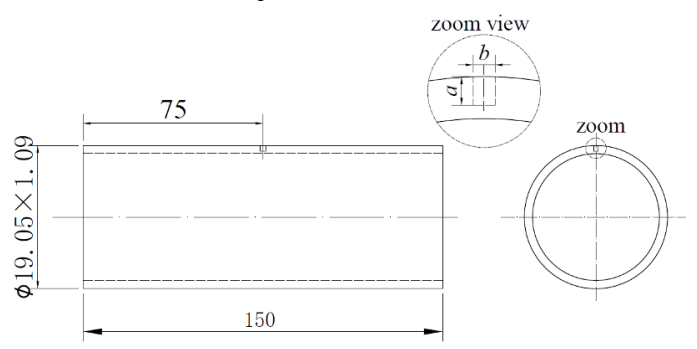

(a) The schematic diagram of heat transfer tube with pit defect.

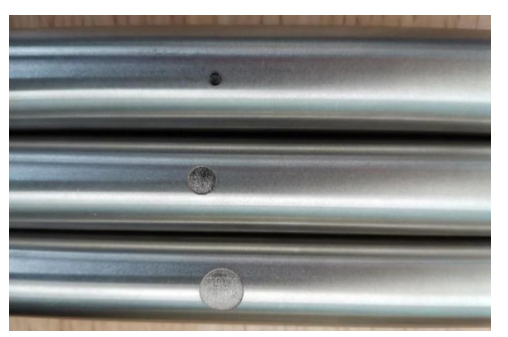

(b) The finished product drawing of heat transfer tube with pit defect.

Figure 1. The specific processing scheme and finished product drawing of artificial injury of sample. 
Table 2. Simulation data of burst pressure of heat transfer tube with defect at $350{ }^{\circ} \mathrm{C}$.

\begin{tabular}{|c|c|c|c|c|c|}
\hline $\begin{array}{l}\text { Sample } \\
\text { number }\end{array}$ & $\begin{array}{c}\text { Diameter } \\
\mathrm{D} / \mathrm{mm}\end{array}$ & $\begin{array}{c}\text { Wall } \\
\text { thickness } \\
t / \mathbf{m m}\end{array}$ & $\begin{array}{c}\text { Actual size } \\
\boldsymbol{a} / \mathbf{m m}\end{array}$ & $\begin{array}{c}\text { Actual size } \\
b / \mathbf{m m}\end{array}$ & $\begin{array}{c}\text { Simulated } \\
\text { burst } \\
\text { pressure } \\
\mathrm{P} / \mathrm{MPa} \\
\end{array}$ \\
\hline (1) & 19.01 & 1.00 & 0.33 & 2.00 & 61.6 \\
\hline (2) & 19.01 & 1.00 & 0.33 & 5.00 & 55.5 \\
\hline (3) & 19.01 & 1.00 & 0.33 & 8.00 & 57.5 \\
\hline (4) & 19.00 & 1.00 & 0.44 & 2.00 & 60.7 \\
\hline (5) & 19.02 & 1.00 & 0.44 & 5.00 & 54.1 \\
\hline (6) & 19.02 & 1.00 & 0.44 & 8.00 & 56.6 \\
\hline (7) & 19.03 & 1.01 & 0.65 & 2.00 & 58.9 \\
\hline (8) & 19.02 & 1.00 & 0.65 & 5.00 & 50.2 \\
\hline (9) & 19.03 & 1.00 & 0.65 & 8.00 & 47.6 \\
\hline
\end{tabular}

\subsection{Simulation data analysis}

Fig. 2 and Fig. 3 show the variation of bursting pressure with the ratio of defect depth and pit diameter, and the damage depth ratio and the diameter of circular defect are the main influencing factors. Fig. 2 shows that the burst pressure decreases with the increase of the damage depth ratio. When the diameter of the defect is small, the bursting pressure has a similar law with the increase of the damage depth ratio. When the diameter of the defect is large, the bursting pressure decreases faster with the increase of the damage depth ratio.

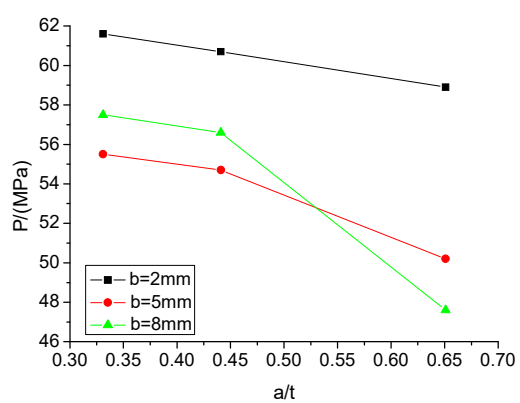

Figure 2. Variation Law of bursting pressure with damage depth ratio $a / t$ under different defect diameter $b$.

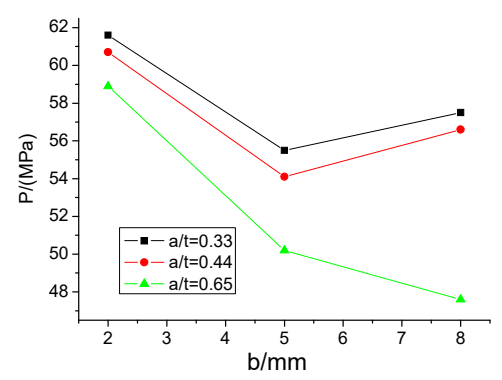

Figure 3. Variation laws of bursting pressure with diameter $b$ of circular pit under different defect depth $\mathrm{a} / \mathrm{t}$.

Fig. 3 shows that the variation of bursting pressure with the diameter of defects shows a complex law under different defect depths. When the damage depth ratio is small, the bursting pressure first decreases and then increases with the increase of defect diameter, and there is a minimum value; when the damage depth ratio is large, the bursting pressure first decreases rapidly and then slowly decreases with the increase of defect diameter. It is mainly because the stress concentration caused by defects can be reduced with the increase of defect diameter. With the increase of diameter, the burst pressure first decreases and then increases. The simulation results show that there is an extremum point of the pit diameter $b$ to maximize the damage for the specimen for a range of depth of defect.

\section{Experimental study on effect of damage characteristics of heat transfer tube on burst pressure}

\subsection{Test equipment and test method}

The high temperature hydraulic burst test method of steam generator heat transfer tube sample is in accordance with the provisions of GB / T 241-2007 "metal tube hydraulic test method". The test is carried out on a high temperature and high pressure testing machine with an environmental chamber, and the constant rate of pressure rise is $13.8 \mathrm{MPa} / \mathrm{min}$. The test device is shown in Fig. 4.

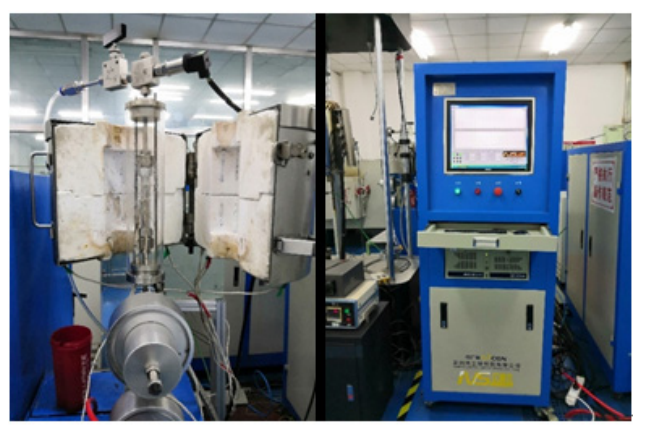

Figure 4. High temperature hydraulic burst test device.

\subsection{The analysis and discussion of test data}

The burst pressure test values of 9 kinds of heat transfer tube samples with defects (numbered as (1), (2), (3), (4), (5), (6), (7), (8) and (9) ) at $350{ }^{\circ} \mathrm{C}$ are listed in Table 3. 
Table 2. Test data of $350^{\circ} \mathrm{C}$ burst pressure for heat transfer tube with defects.

\begin{tabular}{|c|c|}
\hline Sample number & Burst pressure $\boldsymbol{P} / \mathbf{M P a}$ \\
\hline 1 & 61.1 \\
\hline$(2)$ & 54.2 \\
\hline$(3$ & 58.1 \\
\hline$(4)$ & 62.3 \\
\hline (5) & 55.5 \\
\hline 6 & 56.6 \\
\hline 7 & 58.8 \\
\hline 8 & 49.5 \\
\hline (9) & 48.9 \\
\hline
\end{tabular}

Fig. 5 shows the variation law of bursting pressure with defect size of test samples No. (1)-9). Fig. 5a shows the relationship between burst pressure and defect depth ratio $a / t$ when the defect diameter $b$ is $2 \mathrm{~mm}, 5 \mathrm{~m}$ and $8 \mathrm{~mm}$. Fig. $5 \mathrm{~b}$ shows the relationship between burst pressure and defect diameter $b$ when the defect depth ratio a/t is $0.33,0.44$ and 0.65 . Generally speaking, the variation laws of burst pressure with the ratio of damage depth and defect diameter are very complex.

Fig. 5a shows that when the damage depth ratio is small $(a / t \leqslant 0.57)$, the burst pressure of defect diameter $b$ $=5 \mathrm{~mm}$ is lower than that of defect diameter $\mathrm{b}=8 \mathrm{~mm}$, and when the defect diameter increase however the damage is small. When the damage depth ratio is larger $(a / t \geqslant 0.57)$, the burst pressure decreases with the increase of defect diameter b. Fig. $5 b$ shows that when the damage depth ratio is small $(\mathrm{a} / \mathrm{t} \leqslant 0.57)$, there is an extreme point that makes the damage maximum. When the damage depth ratio is large $(a / t \geqslant 0.57)$, the burst pressure decreases with the increase of defect diameter $b$.

The simulation results and test results have similar rules, and the test results verify the simulation results. But the test results and simulation results are also slightly different, the main reason is that the simulation adopts the ideal material model and there are some errors in the test.

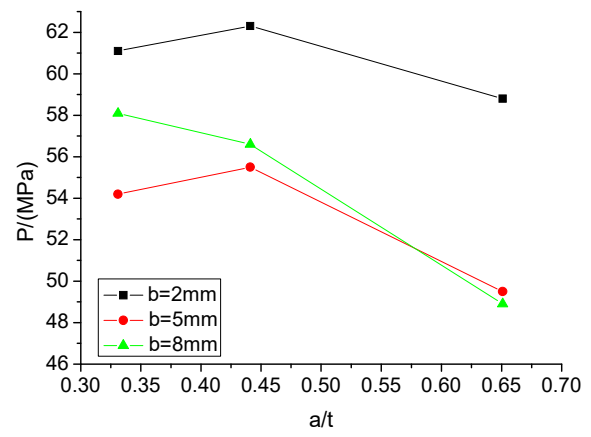

(a) Relationship between burst pressure and defect depth ratio $\mathrm{a} / \mathrm{t}$.

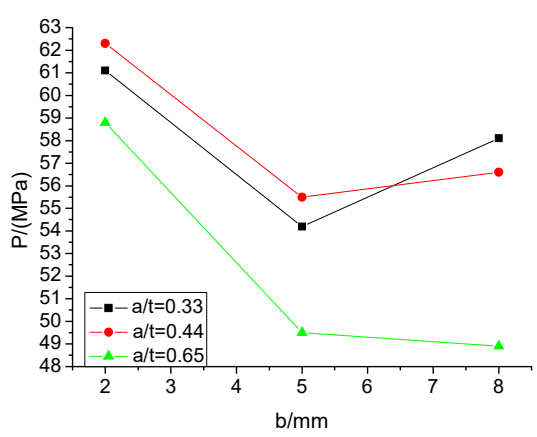

(b) Relationship between burst pressure and defect diameter $b$.

Figure 5. Experimental variation laws of bursting pressure with defect size of specimens.

\section{Conclusions}

Through the simulation analysis and high temperature burst test for inconel690 heat transfer tube sample, the relationship between the size and the bursting pressure of the Inconel690 tube was analyzed. Generally speaking, the variation laws of bursting pressure with the ratio of damage depth and defect diameter are very complex. The results show that:

- When the damage depth ratio is small $(\mathrm{a} / \mathrm{t} \leqslant 0.57)$, the burst pressure of defect diameter of $b=5 \mathrm{~mm}$ is lower than that of defect diameter of $b=8 \mathrm{~mm}$. The smaller the diameter, the greater the damage and the lower the burst pressure,

- When the damage depth ratio is small $(\mathrm{a} / \mathrm{t} \leqslant 0.57)$, The results show that there is an extreme point of maximum damage with the change of defect diameter $b$. With the increase of damage depth ratio $(a / t \geqslant 0.57)$, the burst pressure decreases with the increase of defect diameter $b$, the extreme point of maximum damage disappear.

\section{References}

1. B. Flesch, B. Cochet. Leak-before-break in steam generator tubes, Int J Press Vessels Pip, 43 (1990) 165-179

2. J. H. Lee, Y.W. Park, M.H. Song, Y.J. Kim, S.I. Moon, Determination of equivalent single crack based on coalescence criterion of collinear axial cracks, Nucl. Eng. Des., 205 (2001) 1-11

3. S.S. Hwang, H.P. Kim, J.S. Kim. Evaluation of the burst characteristics for axial notches on SG tubings, Nucl. Eng. Des., 232 (2) (2004) 139-143

4. S.I. Moon, Y.S. Chang, Y.J. Kim, J.H. Lee, M.H. Song, Y.H. Choi, Determination of failure pressure for tubes with two non-aligned through-wall cracks, Int J Fract, 144 (2) (2007) 91-101

5. K.I. Shin, K.B. Yoon, J.H. Park, Experimental and numerical study on the burst pressure of steam generator tubes with 1- and U-Type through-wall combination cracks, J Mech Sci Technol., 27 (8) (2013) 2299-2304 
6. K.H. Eom, J.W. Kim, Y.J. Kim, J.S. Kim, Failure behavior of multiple-axial partthrough-wall flaws in alloy 690TT steam generator tubes, J Press Vessel Technol., 138 (2) (2015) 021406

7. K.H. Lee, H.D. Kim, Y.S. Kang, M.W. Nam, N.C. Cho, Burst pressure tests of axial part-through-wall steam generator tubes, Trans Korean Soc. Press Vessel Pip., 10 (1) (2014) 56-63 